\title{
The role of cytokines in the pathogenesis and staging of Trypanosoma brucei rhodesiense sleeping sickness
}

\author{
Charles D. Kato ${ }^{1}$, Enock Matovu', Claire. M. Mugasa' ${ }^{1}$ Ann Nanteza ${ }^{1}$ and Vincent P. Alibu ${ }^{2 *}$
}

\begin{abstract}
Human African trypanosomiasis due to Trypanosoma brucei rhodesiense is invariably fatal if untreated with up to 12.3 million people at a risk of developing the disease in Sub-Saharan Africa. The disease is characterized by a wide spectrum of clinical presentation coupled with differences in disease progression and severity. While the factors determining this varied response have not been clearly characterized, inflammatory cytokines have been partially implicated as key players. In this review, we consolidate available literature on the role of specific cytokines in the pathogenesis of T. b. rhodesiense sleeping sickness and further discuss their potential as stage biomarkers. Such information would guide upcoming research on the immunology of sleeping sickness and further assist in the selection and evaluation of cytokines as disease stage or diagnostic biomarkers.
\end{abstract}

Keywords: Human African Trypanosomiasis, Cytokines, Immune response, Biomarkers, Sleeping sickness

\section{Background}

Human African trypanosomiasis (HAT) or sleeping sickness is caused by tsetse fly transmitted extra-cellular protozoan parasites Trypanosoma brucei rhodesiense (east and southern Africa) and T. b. gambiense (west and central Africa). It is considered that these species are clinically and epidemiological different thus requiring different therapeutic management [1]. Disease due to $T$. $b$. rhodesiense is classified as acute with rapid progression while T. b. gambiense disease is characterized as chronic $[2,3]$. A reduction in the number of new HAT cases has been reported [4]. However, in endemic areas an estimated 12.3 million people living in or around national parks, forest land and large water bodies are still at a risk of acquiring T. b. rhodesiense disease. More so fatality cases are estimated to be higher than reported since $40 \%$ of cases go undetected and subsequently untreated $[5,6] . T . b$. rhodesiense HAT being a zoonotic disease and

\footnotetext{
*Correspondence: vpalibu@yahoo.com

${ }^{2}$ College of Natural Sciences, Makerere University, P.O. BOX 7062,

Kampala, Uganda

Full list of author information is available at the end of the article
}

endemic in vast areas of continental/tropical Africa [4], elimination cannot be easily achieved.

Previously, T. $b$. rhodesiense HAT has been classified as an acute disease with death occurring within weeks or few months if untreated [2,3]. Recently, a wide spectrum of clinical presentation coupled with differences in disease progression and severity was reported [7-9]. It is now believed that the disease is chronic in south east Africa and progressively more severe and acute towards the north $[8,10]$. It has been demonstrated that individuals from non-endemic areas suffer a more severe disease than those in endemic countries [11, 12]. Furthermore, there seems to be differences in disease progression irrespective of whether the foci are geographically related. A study comparing early stage patients recruited in two geographically distinct areas noted median duration of illness to be longer among Malawi patients (30 days) compared to patients in Uganda (21 days) [8]. In addition, dramatic differences in disease progression and degree of neurological impairment were reported among Ugandan patients in geographically related foci [13]. Subsequently, it is not yet clear if these differences in disease progression and severity are related to the parasite diversity, to host related differences regulating immune responses 
or to both. However, compelling evidence suggests that cytokines might be key players in HAT inflammatory processes $[8,13,14]$.

Reports from animal models and the few studies involving humans suggest that high levels of pro-inflammatory cytokines might be associated with moderate to severe neuropathy $[15,16]$. Furthermore, late stage disease has been associated with elevated levels of counter-inflammatory cytokines in both HAT patients and experimental animal models [16]. Counter-inflammatory cytokines (IL-10 and IL-6) have been associated with a reduction in the severity of neuropathology, suggesting a possible protective role [15]. However, there remains controversy on the role of specific cytokines in disease progression and severity $[17,18]$. In this review, we aim to consolidate available literature on the role of specific cytokines in $T . b$. rhodesiense HAT pathogenesis and to further discuss their potential as stage biomarkers. Such information would guide upcoming research in the immunology of HAT and further assist in the selection and evaluation of cytokines as stage biomarkers and/or develop novel chemotherapeutic interventions.

\section{Diagnosis and diagnostic problems}

Since the clinical signs of HAT are non-specific, in most cases the disease is only suspected in geographical areas where it is endemic. Sleeping sickness is endemic in areas where other tropical diseases like malaria exist [7, 19, 20], making HAT an incidental finding on a blood smear meant for malaria diagnosis. Currently there is an increased bias towards the use of rapid diagnostic tests (RDTs) for the diagnosis of malaria [21]. Therefore, the advent of RDT's for malaria will consequentially lead to reduced detection $T$. $b$. rhodesiense HAT as this relies on the detection of trypanosomes on blood smears. Sleeping sickness occurs in rural sub-Saharan Africa necessitating diagnostic techniques that are simple and cheap to perform [22]. A major constraint in T. b. rhodesiense HAT diagnosis as compared to T. $b$. gambiense HAT is the fact that no suspicion serological tests are yet available thus impairing greatly the detection of cases (both for passive and active detection). Therefore, the most feasible approach for the detection of $T . b$. rhodesiense infections is through direct microscopic observation of trypanosomes in blood, lymph node aspirates or in cerebrospinal fluids (CSF) of highly suspected individuals [23]. Unlike $T$. $b$. gambiense HAT, parasitemia due to T. $b$. rhodesiense is in most cases above the threshold for microscopic detection reaching values of up to 10,000 trypanosomes $/ \mathrm{ml}$ [24]. Thick blood films prepared from a finger prick have limited sensitivity (detection limit is 5000 trypanosomes/ $\mathrm{ml}$ ) but are easy to perform with quick results [25]. In cases of low parasitemia, concentrations/enrichment methods have been used to improve sensitivity. The micro-hematocrit centrifugation technique (mHCT) has a detection limit of 500 trypanosomes/ml $[26,27]$ while the quantitative buffy coat technique offers an improved detection limit of $<500$ trypanosomes/ml [28, 29]. Minianion-exchange centrifugation technique [30] offers an improved sensitivity, detecting $<30$ trypanosomes $/ \mathrm{ml}$ while its improvement on buffy coat goes lower than 10 trypanosomes/ml [31]. Molecular biology techniques that detect parasite nucleic acids with increased sensitivity are becoming more common. The most commonly used PCR technique in research laboratory settings has been the detection of the serum resistance antigen (SRA) to confirm the presence of T. b. rhodesiense [32]. The SRA gene is reported to be responsible for $T$. $b$. rhodesiense human serum resistance but is absent in $T$. $b$. gambiense sub species that is also resistant to lysis by human serum [33]. SRA is able to discriminate T. $b$. rhodesiense from other T. b. brucei sub species with a sensitivity equivalent to 1 trypanosome/ml [34]. Although PCR based techniques have a sensitivity of up to $96 \%$ [35], the techniques have limited application in a field setting due to the need for a thermocycler, power supply and a cold chain for reagents. To improve on their applicability, an isothermal DNA amplification technique called loop mediated isothermal amplification (LAMP) has been developed [36, 37]. LAMP is easier to perform and requires less sophisticated equipment than conventional PCR. However, before its adoption as a diagnostic tool, further clinical validation and standardization on large cohorts is required. Other alternatives like the RNA based real-time nucleic sequence based amplification [38] and oligochromatography-PCR [39] have been developed but are yet to undergo clinical evaluation and validation.

\section{Disease staging}

Since drug treatment for both early and late stage disease differs, it is paramount to accurately determine disease stage. Since it is impossible to stage the disease based on clinical signs, invasive examination of CSF following a lumber puncture is routinely done. Disease stage determination is vital for appropriate treatment. Patients with no trypanosomes in CSF but with a white blood cell count of $\leq 5 \mathrm{WBC} / \mu \mathrm{l}$ are classified as early stage while those with $>5 \mathrm{WBC} / \mu \mathrm{l}$ or with trypanosomes in the CSF are considered late stage patients [23]. With inconsistences and the invasive nature of the current staging method, new stage biomarkers are being proposed [40, 41].

\section{Host immune responses to trypanosomes}

It is now clear that sera from humans and non-human primates has the ability to kill trypanosomes [42, 43]. This ability to kill trypanosomes has been linked to the innate 
trypanosome lytic factors (TLF). Compelling evidence suggests that apolipoprotein LI (ApoLI) and haptoglobinrelated protein ( $\mathrm{Hpr}$ ) might be crucial elements of the TLF $[43,44]$. T. b. brucei has been shown to neutralize the trypanolytic activity of normal human serum through the serum resistance associated protein (SRA) that binds to ApoA1 [32]. Trypanosomes escape host immune recognition through antigenic variation of the membrane bound variant-specific surface glycoprotein, VSG [45]. The VSG acts as a barrier preventing components of the immune responses from accessing the underlying plasma membrane [46]. At peak parasitemia, the parasite releases VSG into circulation [47] thus inducing inflammatory responses. It has been shown that coat switching trypanosomes fail to activate B-cells until coat VSG homogeneity is achieved thus evading recognition [48]. Previously, the main mechanism involved in controlling parasitemia was through antibody production [49-51] with trypanosome specific IgM and IgG reported in the cerebral spinal fluid of late stage patients [52]. In a murine T. b. brucei model using B cell $(\mu \mathrm{MT})$ and IgM-deficient mice, the role of B-cells and IgM antibodies in parasitemia control was investigated [53]. The authors demonstrated that B-cells played a critical role in peak parasitemia clearance while IgM antibodies only played a limited role. However, in another study, a T-cellindependent anti-VSG IgM response was proposed as the first line of defense against proliferating parasites [54]. However, gaps still exist on how and whether antibodies play a significant role in parasite control [55]. Evidence is building up suggesting that cytokines might be key players in HAT pathogenesis $[14,15,56]$.

Most studies on cytokine dysregulations in HAT have used experimental animal models making it possible to follow immunological responses with disease progression [15, 57-61]. Compelling evidence from these studies points to a profound dysregulation in cytokine profiles as a driver for HAT pathogenesis. In general, the early stage of infection is characterized by an elevation in pro-inflammatory cytokines (IFN- $\gamma$ and TNF- $\alpha$ ) with a switch to a counter-inflammatory response in late stage infection $[15$, 62]. It has been demonstrated that prolonged survival to murine African trypanosomiasis might be infection stage dependent, with pro-inflammatory cytokine responses playing a critical role during early stage infection while counter-inflammatory cytokines determine survival during late stage [62]. Furthermore, cytokines in the CNS have been shown to revert to normal levels after treatment making them biomarker candidates for CNS invasion [63, 64]. The role of specific cytokines in HAT progression with emphasis on $T$. $b$. rhodesiense is hereby discussed. The potential roles of cytokines in sleeping sickness and gaps in cytokine research are summarized in Table 1.

Table 1 Potential roles and inconsistencies associated with cytokines in sleeping sickness

\begin{tabular}{|c|c|c|}
\hline Cytokine & Experimental trypanosomiasis & HAT \\
\hline \multicolumn{3}{|l|}{ TNF-a } \\
\hline 1) & Parasite growth control and extended survival $[49,66,109]$ & Associated with rapid disease progression [8] \\
\hline 2) & Control of infection induced pathology $[49,65]$ & No clear role in disease pathogenesis $[71,72]$ \\
\hline 3) & Mediate development of anemia [68] & No data \\
\hline 4) & $\begin{array}{l}\text { Involvement in neuropathology and blood brain barrier dysfunction } \\
{[15,70]}\end{array}$ & No data \\
\hline \multicolumn{3}{|l|}{$\mathrm{IFN}-\gamma$} \\
\hline 1) & Enhance parasite growth [74] & Neurological response involvement [13] \\
\hline 2) & Parasite growth control $[18,61,73]$ & No neurological response involvement [76] \\
\hline 3) & Mediate development of anemia [73] & No data \\
\hline 4) & $\begin{array}{l}\text { Involvement in neuropathology and blood brain barrier dysfunction } \\
{[15,75]}\end{array}$ & No data \\
\hline 5) & Fever induction [60] & No data \\
\hline \multicolumn{3}{|l|}{$\operatorname{IL}-1 \beta$} \\
\hline 1) & Involvement in neuropathology $[15,82,83]$ & No involvement in disease progression or pathology [13] \\
\hline \multicolumn{3}{|l|}{ TGF- $\beta$} \\
\hline 1) & No data & Involvement in pathology [8] No involvement in pathology [13] \\
\hline \multicolumn{3}{|l|}{ IL-6 } \\
\hline 1) & Reduction in neuropathology [15] & No defined role despite elevation in late stage $[13,72,76,92,93]$ \\
\hline \multicolumn{3}{|l|}{ IL-10 } \\
\hline 1) & Reduced pathology and extended survival $[15,61]$ & No defined role despite elevation in late stage $[8,71,72,76,93]$ \\
\hline
\end{tabular}

TNF- $a$ : Tumour necrosis factor- alpha, IFN- $\gamma$ : Interferon gamma, IL-1 $\beta$ : Interleukin-1 beta, TGF- $\beta$ : Transforming growth factor- beta, IL-6: Interleukin-6, IL-10: Interleukin-10, HAT: Human African trypanosomiasis 


\section{Tumour necrosis factor- $a$ (TNF- $a$ )}

Tumour necrosis factor- $\alpha$ (TNF- $\alpha$ ) is a pro-inflammatory cytokine predominantly produced by macrophages and is involved in the innate immunity against intracellular pathogens. Soluble VSGs shed by live trypanosomes are thought to be the major TNF- $\alpha$ inducing factors [65]. To date there is still controversy on the role of TNF- $\alpha$ in HAT infection. Some of the studies using animal models indicate that TNF- $\alpha$ is likely to be a key mediator in the control of T. brucei infections [66-68]. In one study, a direct dose dependent lytic effect of TNF- $\alpha$ on purified T. b. gambiense parasites was reported suggesting an involvement in parasite growth control [69]. However, detrimental roles of TNF- $\alpha$ have also been reported. In a murine model, TNF- $\alpha$ knockout mice exhibited trypanosome-mediated immunopathological features such as, lymphnode associated immunosuppression and lipopolysaccharide hypersensitivity [67]. High levels of brain TNF- $\alpha$ were associated with moderate to severe neuropathy [15]. In a T. b. rhodesiense vervet monkey model, CNS TNF- $\alpha$ levels did not differ from controls and no association with clinical presentation was reported [60]. Furthermore, it has been demonstrated in a murine model that TNF- $\alpha$ might be involved in anemia associated with T. b. rhodesiense infections and not in T. congolense [68]. This demonstrates the challenges in comparing studies utilizing different trypanosome or host species. There is evidence pointing to a possible role of TNF- $\alpha$ in trypanosome penetration of the blood brain barrier, especially through Toll-like receptor (TLR)-MyD88-mediated signaling [70]. Human T. b. rhodesiense studies testing predictions from experimental animal models have started to emerge. A study comparing plasma cytokine levels between geographically isolated HAT foci reported elevated levels of TNF- $\alpha$ in Ugandan patients as compared to their counterparts in Malawi [8]. The author's proposed that TNF- $\alpha$ might play a role in the rapid disease progression reported among Uganda patients. On the contrary, in another study in Uganda both plasma and CSF TNF- $\alpha$ levels remained at baseline [71], just as previously reported among $T$. b. gambiense patients [72]. From available literature for both animal models and human studies, the role of TNF- $\alpha$ in $T . b$. rhodesiense disease pathology remains largely unclear.

\section{Interferon gamma (IFN- $\gamma$ )}

Interferon gamma is a pro-inflammatory cytokine secreted primarily by $\mathrm{T}$ - and natural killer (NK) cells with a role in innate immunity and also as an inducer of the adaptive immune response. The role of IFN- $\gamma$ in HAT progression has been investigated using experimental animal models and in few human studies. In a murine T. $b$. brucei model, IFN- $\gamma$ knockout mice suffered uncontrolled parasitemia with a significant reduction in survival time compared to the wild type mice [61, 73]. Similar observations were made in a $T . b$. rhodesiense murine model in which IFN- $\gamma$ was associated with a decrease in parasite numbers and resistance to infection [18]. These findings show that IFN- $\gamma$ might be essential in parasite control. On the contrary, in a study utilizing mononuclear cell cultures, rat IFN- $\gamma$ was associated with an increase in parasite numbers [74]. Furthermore, in a murine T. b. brucei model an exponential increase in the severity of the neurological response was associated with increased levels of brain IFN- $\gamma$ [15]. IFN- $\gamma$ was further demonstrated to be a prerequisite for $T$. b. brucei parasite transmigration across the blood brain barrier (BBB) [75]. In a T. b. rhodesiense vervet monkey model, serum IFN- $\gamma$ was upregulated in the infected group with a positive correlation to body temperature during the early phase of disease. In this model, IFN- $\gamma$ was never detected in CSF up to day 42 post-infection [60]. Similarly, in human patients, serum IFN- $\gamma$ was significantly elevated in early stage patients compared to late stage patients and for both stages higher than control levels [71]. In another related T. $b$. rhodesiense sleeping sickness study comparing CSF cytokine levels between two geographically similar HAT foci (Soroti and Tororo), elevated IFN- $\gamma$ levels were associated with moderate to severe comma [13]. This study provided for the first time, clinical evidence that IFN- $\gamma$ might be associated with clinical signs of neurological involvement in T. $b$. rhodesiense HAT. In a related study in Eastern Uganda, when CSF IFN- $\gamma$ concentrations were compared across disease stage no significant differences were noted [76]. Moreover, in the latter study no significant association was noted regarding presence or absence of neuropathology with IFN- $\gamma$ levels. Within the current literature, the role of IFN- $\gamma$ in T. $b$. rhodesiense disease remains uncertain.

\section{Interleukin-1 beta (IL-1 $\beta$ )}

Interleukin-1 beta is a pro-inflammatory cytokine belonging to IL-1 family of cytokines [77]. It is produced mainly by monocytes and macrophages [78]. In a number of inflammatory disorders, IL- $1 \beta$ has been associated with both innate and adaptive immune responses $[79,80]$, with a possible role in BBB dysfunction [81]. However, its role in HAT, is not well defined. In a murine model utilizing a T. b. brucei cloned stabilate (GVR35/ C1.8), there was an apparent increase in plasma levels of IL- $1 \beta$ but not significantly different from controls [15]. In this study, IL-1 $\beta$ levels were not correlated to the degree of neuro-inflammation. However, in a stepwise multiple linear regression analysis, it was noted that IL-1 $\beta$, 
TNF- $\alpha$ and IFN- $\gamma$ levels in the brain accounted for over $94.8 \%$ of the variation in neuropathology. Furthermore, intraventricular injection of an IL-1 receptor antagonist together with sTNF-r1 antagonist augment the reduction in neurodegeneration caused by trypanosome infection compared with infusion of sTNF-r1 antagonist alone [82]. In a T. b. brucei murine model, mRNA transcripts for IL- $1 \beta$ were localized in areas showing apoptosis and nerve fiber degeneration [83]. In this study, neuropathology was not solely attributed to IL-1 $\beta$. A study involving T. b. rhodesiense HAT patients in two Ugandan foci (Tororo and Soroti) reported no significant differences in plasma IL- $1 \beta$ levels despite variations in disease progression and severity between the two foci [13]. In this study, the authors were unsuccessful in detecting IL- $1 \beta$ in the CSF. Available literature does not clearly define the role played by IL- $1 \beta$ in HAT pathogenesis.

\section{Transforming growth factor-beta (TGF- $\beta$ )}

Transforming growth factor- beta is a pluripotent cytokine with both pro- and counter-inflammatory effects depending on its environment and concentration [84]. At higher concentrations, TGF- $\beta$ is reported to play an immuno-modulatory role through the suppression of TNF- $\alpha$ and IFN- $\gamma$ synthesis by peripheral blood mononuclear cells and peritoneal-derived macrophages [85]. In HAT, literature on the role of TGF- $\beta$ in disease progression is scanty. Nevertheless, TGF- $\beta$ has been proposed to influence pathogenesis of $T$. $b$. rhodesiense sleeping sickness. A study comparing T. $b$. rhodesiense cytokine profiles in two geographically distinct HAT foci (Uganda and Malawi) reported a significant increase in plasma TGF- $\beta$ levels in Malawi patients as compared to patients in Uganda [8]. The authors argued that the higher levels of TGF- $\beta$ in plasma of Malawi patients might be responsible for the reduced pathology and prolonged survival in this group. On the contrary, Maclean et al. [13] comparing plasma TGF- $\beta$ levels in two HAT foci in Uganda did not find significant difference despite the disease being more severe in Tororo compared to Soroti. In this study plasma TGF- $\beta$ levels were significantly higher in HAT patients than controls. These findings suggest an involvement of TGF- $\beta$ in HAT pathogenesis though its specific role is not clearly understood. In experimental animal models, the role of TGF- $\beta$ in trypanosomiasis has not been investigated making results from human studies difficult to interpret. Animal models might be helpful in refining the general observations made in human studies.

\section{Interleukin-6 (IL-6)}

Interleukin- 6 is a multi-functional cytokine shown to possess both pro- and counter-inflammatory effects with varied implications in pathophysiology of many neurological and inflammatory disorders. In other disorders, IL-6 was shown to possess beneficial effects involving metabolic control [86], neuronal survival [87], neuro-protective and analgesic effects in rats [88]. On the other hand, destructive properties have also been reported. IL-6 has been associated with neuronal degeneration and cell death in degenerative disorders [89]. Furthermore, in other neuropathological disorders, mice over expressing IL-6 were associated with increased BBB permeability coupled with neuropathological abnormalities [90]. In a murine T. b. brucei model, high levels of IL-6 were observed in mice with less severe neuropathology [15]. These findings were consistent with studies in a T. b. rhodesiense vervet monkey model in which CSF IL-6 levels were up regulated in late stage disease [91]. Similarly, in HAT patients CSF IL-6 was upregulated in late stage T. b. rhodesiense disease [76, 92] and in T. b. gambiense disease [72, 93]. However, in all these studies, the role of IL-6 in HAT pathogenesis was not investigated. A study comparing plasma levels of IL-6 in two HAT foci in Uganda reported higher levels in Soroti with mild disease as compared to Tororo with a more acute disease [13]. The implication for the elevated IL-6 levels in Soroti patients were not explained. However, it is possible that IL-6 plays a protective role as reported in experimental animals. Consequently, although murine models point to a protective role, in humans the role of IL- 6 cannot yet be clearly defined.

\section{Interleukin-10 (IL-10)}

IL-10 is a regulatory cytokine that is produced presumably to control excessive inflammation by a variety of cell types within the innate and adaptive immune systems including macrophages, T- and B-cells [94]. IL-10 has been demonstrated to upregulate the production of antibodies and elevate MHC class II expression on B cells [95]. In a number of parasitic diseases, IL-10 has been shown to possess host protective roles, including malaria [96], toxoplasmosis and in autoimmune encephalitis [97]. Similar roles of IL10 have been described in T. brucei experimental murine models.

In one study, the absence of IL-10 in wild type mice was associated with decreased survival in $T . b$. brucei infected mice [61]. Furthermore, mice with increased IL-10 levels were associated with markedly reduced IFN- $\gamma$ concentrations and subsequently survived longer than infected control animals. The authors thus suggested that IL-10 might play a role in providing a balance between pathogenic and protective immune response during $T . b$. bru$c e i$ infection [61]. Furthermore, in another murine model using T. $b$. brucei cloned stabilate, mice with elevated CNS IL-10 levels were associated with mild inflammatory pathology [15]. In HAT, plasma and CNS levels of 
IL-10 were upregulated particularly in the late stage of both T. $b$. rhodesiense $[8,71]$ and in T. b. gambiense $[72,93]$. However, in all these studies the role of IL-10 on HAT associated pathology was not investigated. In a study by Maclean et al. [76] among T. $b$. rhodesiense patients in Uganda, CSF IL-10 did not significantly associate with neurological signs of ataxia, tremors, or urinary incontinence. Likewise, a related study comparing plasma cytokine profiles in two foci in Uganda (Tororo and Soroti) did not find a significant difference in IL-10 levels despite the disease being more severe in Tororo. Notwithstanding, even if $T . b$. rhodesiense sleeping sickness has been associated with dysregulation in IL-10 levels, its significance in disease pathogenesis has not been clearly demonstrated.

\section{Cytokines as potential stage biomarkers}

According to a biomarker working group [98], a biomarker is an objectively measured and evaluated characteristic indicating a physiological process, pathogenic process or pharmacological response to a therapeutic intervention. The current HAT staging criteria rely on WBC counting and the detection of parasites in CSF. However, in some cases these criteria have been shown to give false results since trypanosomes are not usually detected in CSF and an elevation in white blood cells is not necessarily specific to trypanosomes [99, 100]. Furthermore, the need for using a lumber puncture to obtain CSF is invasive, a discomfort to the patient and requires trained personnel. Due to these shortfalls in the current staging, a number of novel biomarkers are currently being sought. With the observation that specific cytokines are upregulated in late stage sleeping sickness, they have been proposed as potential stage biomarkers [76, 91]. Among the cytokines, IL-10 and IL-6 have shown greater potential due to their association with late stage disease in the CNS. Additionally, both plasma and CSF IL-10 levels were reported to return to normal following treatment in a $T . b$. rhodesiense vervet monkey model [56], in T. b. gambiense sleeping sickness [101] and in T. b. rhodesiense sleeping sickness [71]. From these finding, IL-10 was proposed as a marker for cure. However, despite these promising observations, data from clinical evaluation of these markers is limited. In a T. $b$. rhodesiense study, CSF IL-10 was upregulated in late stage patients [76]. When its utility as a potential late stage marker was evaluated, CSF IL-10 was insufficiently sensitive detecting only 14 out of 100 late stage patients [76]. In a more recent study, a higher staging accuracy for both CSF IL- 6 and IL-10 was reported [102]. From this study, at a specificity of $100 \%$, IL-10 would detect around 86 out of 100 late stage patients while IL-6 detected around 83 out of 100 late stage patients. However, in this study the authors acknowledged the fact that most patients were diagnosed as late stage hence limiting comparisons with the few early stage patients. Tiberti et al. [100] demonstrated that when $T$. $b$. rhodesiense biomarkers are used as a panel, sensitivity and specificity is greatly enhanced. A number of other promising markers distinguishing between early and late stage patients have been proposed including; chemokines and the heart-fatty acid binding protein $[41,100,103]$, immunoglobulins $[56,101]$, cell adhesion molecules [104], neopterin [105], osteopontin and $\beta 2$ microglobulin [106] and matrix metalloproteinase-9 [104] and recently neuronal specific enolase [107]. Indeed, when CSF IL-10 was evaluated as a panel with TGF- $\beta$ and IgM, the panel had an improved sensitivity, from detecting 14 out of 100 late stage patients to detecting 70 out of 100 late stage cases [76]. However, to date literature about the use of cytokines as panels to improve staging accuracy is scanty and therefore does not allow meaningful comparisons.

Basing on available literature, the possibility of translating cytokines into point-of-care tests for stage determination has some draw backs. Firstly, cytokine markers are not $100 \%$ sensitive and their application might lead to wrong treatment choices that could lead to relapses since some late stage patients would be missed. In order to improve on sensitivity, studies analyzing cytokines as panels or in combination with other previously identified markers might be helpful. Secondly, the need to rely on the invasive collection of CSF by lumber puncture makes the direct field application of CSF cytokines and other novel markers problematic. To date no plasma cytokine has shown potential as a stage biomarker. To better assess the value of plasma cytokines, studies utilizing larger sample size representative of the population at risk and tools such as Luminex Chips that enable the quantification in a single sample of large numbers of cytokines or proteomic approaches such as the one performed on the CSF are required. Lastly, sleeping sickness is endemic in areas were other tropical diseases are common [7]. This is complicated by the fact that cytokine dysregulations and biomarker potential apply to other CNS disorders [108], thus clouding direct interpretation of cytokine data. Therefore, clinical validation of cytokine data in light of other co-infections would be helpful in identifying specific cytokines that might be unique to HAT.

\section{Conclusions}

Although literature on cytokine dysregulation in $T$. $b$. rhodesiense HAT is scarce, it is quite clear that high levels of pro-inflammatory cytokines are associated with immunopathology. However, in late stage disease, an elevation in counter-inflammatory cytokines is associated 
with a reduction in the degree of immunopathology. In some cases, inconsistences about the role of specific cytokines in HAT pathogenesis have been documented. This points to the fact that pathogenesis might be influenced by a complex interaction of cytokines with many having multiple roles. Moreover, due to ethical considerations in human studies, serial measurements of cytokines with disease progress have not been done making a clear distinction between cause and effect roles for the specific cytokines problematic. Nevertheless, cytokines have been proposed as potential diagnostic or stage biomarkers. Indeed, due to their up-regulation in late stage diseases, counter-inflammatory cytokines including IL-10, IL-6 and TGF- $\beta$ were proposed as stage biomarkers. However, before cytokines can be considered as biomarkers, more clinical studies are required for validation. On the other hand, though murine models have provided invaluable information, inconsistences from human studies have been reported. Therefore, extrapolating data from these models might give erroneous conclusions about cytokine roles in HAT. Recently, the vervet monkey model has been shown to develop disease clinically and immunologically similar to T. b. rhodesiense HAT in humans [91] and would be helpful in refining cytokine roles with disease progression.

\begin{abstract}
Abbreviations
BBB: blood brain barrier; CNS: central nervous system; CSF: cerebrospinal fluid; HAT: human African trypanosomiasis; IFN: interferon; IL: interleukin; LAMP: loop mediated isothermal amplification; MGE: mobile genetic element; mHCT: mini-anion-exchange centrifugation technique; PCR: polymerase Chain Reaction; RAPD: random amplified polymorphism DNA; RDTs: rapid diagnostic tests; SRA: serum resistance antigen; TGF- $\beta$ : transforming growth factor- beta; TNF: tumor necrosis factor; WBC: white blood cell; WHO: World Health Organization.
\end{abstract}

\section{Authors' contributions}

VPA \& EM received the grant; CDK, VPA \& EM conceived the write up; CDK, AN and CM wrote the paper; EM \& VPA reviewed the paper. All authors read and approved the final manuscript.

\section{Author details \\ ${ }^{1}$ School of Bio-security, Biotechnical \& Laboratory Sciences, College of Veteri- nary Medicine, Animal Resources \& Bio-security, Makerere University, P.O BOX 7062, Kampala, Uganda. ${ }^{2}$ College of Natural Sciences, Makerere University, P.O. BOX 7062, Kampala, Uganda.}

\section{Acknowledgements}

Our work was carried out within the framework of the consortium Afrique One "Ecosystem and Population Health: Expanding Frontiers in Health" (http://www.afriqueone.net/). Afrique One is funded by the Wellcome Trust (087535/Z/08/A). We thank the Consortium for Advanced Research Training in Africa (CARTA) through the ESEO program for their support with writing.

\section{Competing interests}

The authors declare that they have no competing interests.

Received: 20 October 2015 Accepted: 6 January 2016

Published online: 22 January 2016

\section{References}

1. Welburn SC, Fèvre EM, Coleman PG, Odiit M, Maudlin I. Sleeping sickness: a tale of two diseases. Trends Parasitol. 2001;17(1):19-24.

2. Barrett MP, Burchmore RJ, Stich A, Lazzari JO, Frasch AC, Cazzulo JJ, et al. The trypanosomiases. Lancet. 2003;362(9394):1469-80.

3. Foulkes J. The six diseases WHO. Human trypanosomiasis in Africa. Br Med J. 1981;283(6300):1172-4

4. Simarro PP, Cecchi G, Franco JR, Paone M, Diarra A, Ruiz-Postigo JA, et al. Estimating and mapping the population at risk of sleeping sickness. PLoS Negl Trop Dis. 2012;6(10):e1859. doi:10.1371/journal. pntd.0001859.

5. Matemba LE, Fèvre EM, Kibona SN, Picozzi K, Cleaveland S, Shaw AP, et al. Quantifying the burden of rhodesiense sleeping sickness in Urambo District, Tanzania. PLoS Negl Trop Dis. 2010;4(11):e868.

6. Odiit M, Coleman P, Liu WC, McDermott J, Fevre E, Welburn S, et al. Quantifying the level of under-detection of Trypanosoma brucei rhodesiense sleeping sickness cases. Trop Med Int Health. 2005;10(9):840-9.

7. Kato CD, Nanteza A, Mugasa C, Edyelu A, Matovu E, Alibu VP. Clinical profiles, disease outcome and co-morbidities among T.b. rhodesiense sleeping sickness patients in Uganda. PLoS One. 2015;10(2):e0118370.

8. MacLean LM, Chisi JE, Odiit M, Gibson WC, Ferris V, Picozzi K, et al. Severity of human African trypanosomiasis in East Africa is associated with geographic location, parasite genotype, and host inflammatory cytokine response profile. Infect Immun. 2004;72(12):7040-4.

9. MacLean LM, Odiit M, Chisi JE, Kennedy PG, Sternberg JM. Focusspecific clinical profiles in human African trypanosomiasis caused by Trypanosoma brucei rhodesiense. PLoS Negl Trop Dis. 2010;4(12):e906

10. Ormerod W. Taxonomy of the sleeping sickness trypanosomes. The Journal of parasitology. 1967:824-30.

11. Blum J, Neumayr A, Hatz C. Human African trypanosomiasis in endemic populations and travellers. Eur J Clin Microbiol Infect Dis. 2012;31(6):905-13.

12. Duggan AJ, Hutchinson MP. Sleeping sickness in Europeans: a review of 109 cases. J Trop Med Hyg. 1966;69(6):124-31.

13. MacLean LM, Odiit M, MacLeod A, Morrison L, Sweeney L, Cooper A, et al. Spatially and genetically distinct African Trypanosome virulence variants defined by host interferon- $\gamma$ response. J Infect Dis. 2007;196(11):1620-8.

14. Sternberg JM, MacLean L. A spectrum of disease in human African trypanosomiasis: the host and parasite genetics of virulence. Parasitology. 2010;137(14):2007-15.

15. Sternberg JM, Rodgers J, Bradley B, MacLean L, Murray M, Kennedy PG. Meningoencephalitic African trypanosomiasis: brain IL-10 and IL-6 are associated with protection from neuro-inflammatory pathology. J Neuroimmunol. 2005;167(1):81-9.

16. Kennedy PG. Cytokines in central nervous system trypanosomiasis: cause, effect or both? Trans R Soc Trop Med Hyg. 2009;103(3):213-4.

17. Bakhiet M, Olsson T, Mhlanga J, Buscher P, Lycke N, van der Meide PH, et al. Human and rodent interferon-gamma as a growth factor for Trypanosoma brucei. Eur J Immunol. 1996;26(6):1359-64. doi:10.1002/ eji.1830260627.

18. Hertz CJ, Filutowicz H, Mansfield JM. Resistance to the African trypanosomes is IFN-gamma dependent. J immunol (Baltimore, Md: 1950). 1998;161(12):6775-83.

19. Kagira JM, Maina N, Njenga J, Karanja SM, Karori SM, Ngotho JM. Prevalence and types of coinfections in sleeping sickness patients in kenya (2000/2009). J Trop Med. 2011;2011:248914. doi:10.1155/2011/248914.

20. Kuepfer I, Hhary EP, Allan M, Edielu A, Burri C, Blum JA. Clinical presentation of $\mathrm{Tb}$ rhodesiense sleeping sickness in second stage patients from Tanzania and Uganda. PLoS Negl Trop Dis. 2011;5(3):e968.

21. Gillet P, Maltha J, Hermans V, Ravinetto R, Bruggeman C, Jacobs J. Malaria rapid diagnostic kits: quality of packaging, design and labelling of boxes and components and readability and accuracy of information inserts. Malar J. 2011;10:39. doi:10.1186/1475-2875-10-39.

22. Mitashi P, Hasker E, Lejon V, Kande V, Muyembe JJ, Lutumba P, et al. Human african trypanosomiasis diagnosis in first-line health services of endemic countries, a systematic review. PLoS Negl Trop Dis. 2012;6(11):e1919. doi:10.1371/journal.pntd.0001919. 
23. WHO. Control and surveillance of African trypanosomiasis. Report of a WHO Expert Committee. World Health Organ Tech Rep Ser. 1998;881:1114 (I-VI).

24. Brun R, Blum J, Chappuis F, Burri C. Human African trypanosomiasis. Lancet. 2010;375(9709):148-59.

25. Miezan TW, Meda AH, Doua F, Cattand P. Evaluation of the parasitologic technics used in the diagnosis of human Trypanosoma gambiense trypanosomiasis in the Ivory Coast. Bull soc Pathol Exot (1990). 1994;87(2):101-4

26. Ancelle T, Paugam A, Bourlioux F, Merad A, Vigier JP. Detection of trypanosomes in blood by the Quantitative Buffy Coat (QBC) technique: experimental evaluation. Med Trop (Mars). 1997;57(3):245-8.

27. Truc P, Jamonneau V, N'Guessan P, Diallo P, Garcia A. Parasitological diagnosis of human African trypanosomiasis: a comparison of the $\mathrm{QBC}^{\circledR}$ and miniature anion-exchange centrifugation techniques. Trans R Soc Trop Med Hyg. 1998;92(3):288-9.

28. Woo PT. The haematocrit centrifuge technique for the diagnosis of African trypanosomiasis. Acta Trop. 1970;27(4):384-6.

29. Woo PT. Evaluation of the haematocrit centrifuge and other techniques for the field diagnosis of human trypanosomiasis and filariasis. Acta Trop. 1971;28(3):298-303.

30. Lumsden W, Kimber C, Evans D, Doig S. Trypanosoma brucei: miniature anion-exchange centrifugation technique for detection of low parasitaemias: adaptation for field use. Trans R Soc Trop Med Hyg. 1979;73(3):312-7.

31. Camara M, Camara O, Ilboudo H, Sakande H, Kaboré J, N’Dri L, et al. Sleeping sickness diagnosis: use of buffy coats improves the sensitivity of the mini anion exchange centrifugation test. Trop Med Int Health. 2010;15(7):796-9.

32. Van Xong H, Vanhamme L, Chamekh M, Chimfwembe CE, Van Den Abbeele J, Pays A, et al. A VSG expression site-associated gene confers resistance to human serum in Trypanosoma rhodesiense. Cell. 1998;95(6):839-46.

33. De Greef C, Imberechts H, Matthyssens G, Van Meirvenne N, Hamers R. A gene expressed only in serum-resistant variants of Trypanosoma brucei rhodesiense. Mol Biochem Parasitol. 1989;36(2):169-76.

34. Radwanska M, Chamekh M, Vanhamme L, Claes F, Magez S, Magnus $E$, et al. The serum resistance-associated gene as a diagnostic tool for the detection of Trypanosoma brucei rhodesiense. Am J Trop Med Hyg. 2002;67(6):684-90.

35. Jamonneau V, Solano P, Garcia A, Lejon V, Dje N, Miezan T, et al. Stage determination and therapeutic decision in human African trypanosomiasis: value of polymerase chain reaction and immunoglobulin $\mathrm{M}$ quantification on the cerebrospinal fluid of sleeping sickness patients in Cote d'Ivoire. Trop Med Int Health. 2003;8(7):589-94.

36. Kuboki N, Inoue N, Sakurai T, Di Cello F, Grab DJ, Suzuki H, et al. Loopmediated isothermal amplification for detection of African trypanosomes. J Clin Microbiol. 2003;41(12):5517-24.

37. Njiru ZK, Mikosza ASJ, Armstrong T, Enyaru JC, Ndung'u JM, Thompson ARC. Loop-mediated isothermal amplification (LAMP) method for rapid detection of Trypanosoma brucei rhodesiense. PLoS Negl Trop Dis. 2008;2(2):e147.

38. Mugasa CM, Laurent T, Schoone GJ, Kager PA, Lubega GW, Schallig HD. Nucleic acid sequence-based amplification with oligochromatography for detection of Trypanosoma brucei in clinical samples. J Clin Microbiol. 2009:47(3):630-5.

39. Deborggraeve S, Claes F, Laurent T, Mertens P, Leclipteux T, Dujardin J, et al. Molecular dipstick test for diagnosis of sleeping sickness. J Clin Microbiol. 2006;44(8):2884-9.

40. Amin DN, Ngoyi DM, Nhkwachi G-M, Palomba M, Rottenberg M, Büscher $P$, et al. Identification of stage biomarkers for human African trypanosomiasis. Am J Trop Med Hyg. 2010;82(6):983-90.

41. Hainard A, Tiberti N, Robin X, Lejon V, Ngoyi DM, Matovu E, et al. A combined CXCL10, CXCL8 and H-FABP panel for the staging of human African trypanosomiasis patients. PLoS Negl Trop Dis. 2009;3(6):e459.

42. Molina-Portela MP, Samanovic M, Raper J. Distinct roles of apolipoprotein components within the trypanosome lytic factor complex revealed in a novel transgenic mouse model. J Exp Med. 2008;205(8):1721-8.

43. Vanhollebeke B, Truc P, Poelvoorde P, Pays A, Joshi PP, Katti R, et al. Human Trypanosoma evansi infection linked to a lack of apolipoprotein L-I. N Engl J Med. 2006;355(26):2752-6.
44. Vanhollebeke B, Nielsen MJ, Watanabe Y, Truc P, Vanhamme L, Nakajima $\mathrm{K}$, et al. Distinct roles of haptoglobin-related protein and apolipoprotein L-I in trypanolysis by human serum. Proc Natl Acad Sci USA. 2007;104(10):4118-23.

45. Pays $E$. The variant surface glycoprotein as a tool for adaptation in African trypanosomes. Microbes Infect. 2006;8(3):930-7.

46. Ferrante A, Allison A. Alternative pathway activation of complement by African trypanosomes lacking a glycoprotein coat. Parasite Immunol. 1983;5(5):491-8.

47. Black S, Hewett RS. Trypanosoma brucei variable surface antigen is released by degenerating parasites but not by actively dividing parasites. Parasite Immunol. 1982;4(4):233-44.

48. Dubois ME, Demick KP, Mansfield JM. Trypanosomes expressing a mosaic variant surface glycoprotein coat escape early detection by the immune system. Infect Immun. 2005;73(5):2690-7.

49. Magez S, Stijlemans B, Baral T, De Baetselier P. VSG-GPI anchors of African trypanosomes: their role in macrophage activation and induction of infection-associated immunopathology. Microbes Infect. 2002;4(9):999-1006.

50. Mansfield JM. Immunobiology of African trypanosomiasis. Cell Immunol. 1978;39(1):204-10.

51. Pentreath $\mathrm{V}$. Trypanosomiasis and the nervous system: pathology and immunology. Trans R Soc Trop Med Hyg. 1995;89(1):9-15.

52. Lejon V, Büscher P, Magnus E, Moons A, Wouters I, Van Meirvenne N. A semi-quantitative ELISA for detection of Trypanosoma brucei gambiense specific antibodies in serum and cerebrospinal fluid of sleeping sickness patients. Acta Trop. 1998;69(2):151-64.

53. Magez S, Schwegmann A, Atkinson R, Claes F, Drennan M, De Baetselier $\mathrm{P}$, et al. The role of B-cells and IgM antibodies in parasitemia, anemia, and VSG switching in Trypanosoma brucei-infected mice. PLoS Pathog. 2008:4(8):e1000122

54. Reinitz DM, Mansfield JM. T-cell-independent and T-cell-dependent B-cell responses to exposed variant surface glycoprotein epitopes in trypanosome-infected mice. Infect Immun. 1990;58(7):2337-42.

55. Stijlemans B, Guilliams M, Raes G, Beschin A, Magez S, De Baetselier P. African trypanosomosis: from immune escape and immunopathology to immune intervention. Vet Parasitol. 2007;148(1):3-13.

56. Ngotho M, Kagira J, Jensen H, Karanja S, Farah I, Hau J. Immunospecific immunoglobulins and IL-10 as markers for Trypanosoma brucei rhodesiense late stage disease in experimentally infected vervet monkeys. Trop Med Int Health. 2009;14(7):736-47.

57. Hertz CJ, Mansfield JM. IFN- $\gamma$-dependent nitric oxide production is not linked to resistance in experimental African trypanosomiasis. Cell Immunol. 1999;192(1):24-32.

58. Hunter CA, Jennings FW, Kennedy PG, Murray M. Astrocyte activation correlates with cytokine production in central nervous system of Trypanosoma brucei brucei-infected mice. Lab Invest. 1992;67(5):635-42.

59. Kaushik RS, Uzonna JE, Zhang Y, Gordon JR, Tabel H. Innate resistance to experimental African trypanosomiasis: differences in cytokine (TNF-a, $\mathrm{IL}-6, \mathrm{IL}-10$ and IL-12) production by bone marrow-derived macrophages from resistant and susceptible mice. Cytokine. 2000;12(7):1024-34.

60. Maina N, Ngotho JM, Were T, Thuita JK, Mwangangi DM, Kagira JM, et al. Proinflammatory cytokine expression in the early phase of Trypanosoma brucei rhodesiense infection in vervet monkeys (Cercopithecus aethiops). Infect Immun. 2004;72(5):3063-5.

61. Namangala B, Noël W, De Baetselier P, Brys L, Beschin A. Relative contribution of interferon- $\gamma$ and interleukin-10 to resistance to murine African trypanosomosis. J Infect Dis. 2001;183(12):1794-800.

62. Namangala B, De Baetselier P, Beschin A. Both type-I and type-II responses contribute to murine trypanotolerance. J Vet Med Sci. 2009;71(3):313-8.

63. Bucheton B, MacLeod A, Jamonneau V. Human host determinants influencing the outcome of Trypanosoma brucei gambiense infections. Parasite Immunol. 2011;33(8):438-47.

64. Rhind SG, Sabiston BH, Shek PN, Buguet A, Muanga G, Stanghellini A, et al. Effect of melarsoprol treatment on circulating IL-10 and TNF-a levels in human African trypanosomiasis. Clin Immunol Immunopathol. 1997:83(2):185-9.

65. Magez S, Stijlemans B, Radwanska M, Pays E, Ferguson MA, De Baetselier P. The glycosyl-inositol-phosphate and dimyristoylglycerol moieties of the glycosylphosphatidylinositol anchor of the trypanosome 
variant-specific surface glycoprotein are distinct macrophage-activating factors. J Immunol (Baltimore, Md: 1950). 1998;160(4):1949-56.

66. Magez S, Geuskens M, Beschin A, Del Favero H, Verschueren H, Lucas R, et al. Specific uptake of tumor necrosis factor-a is involved in growth control of Trypanosoma brucei. J Cell Biol. 1997;137(3):715-27.

67. Magez S, Radwanska M, Beschin A, Sekikawa K, De Baetselier P. Tumor necrosis factor alpha is a key mediator in the regulation of experimental Trypanosoma brucei infections. Infect Immun. 1999;67(6):3128-32.

68. Naessens J, Kitani H, Nakamura Y, Yagi Y, Sekikawa K, Iraqi F. TNF-a mediates the development of anaemia in a murine Trypanosoma brucei rhodesiense infection, but not the anaemia associated with a murine Trypanosoma congolense infection. Clin Exp Immunol. 2005;139(3):405-10.

69. Dauloue'd S, Bouteille B, Moynet D, Lemesre J, Buguet A, Cespuglio R, et al. Human macrophage tumor necrosis factor (TNF)-a production induced by Trypanosoma brucei gambiense and the Role of TNF-a in parasite control. J Infect Dis. 2001;183:988-91.

70. Amin DN, Vodnala SK, Masocha W, Sun B, Kristensson K, Rottenberg ME. Distinct toll-like receptor signals regulate cerebral parasite load and

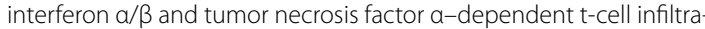
tion in the brains of Trypanosoma brucei-Infected Mice. J Infect Dis 2012;205(2):320-32. doi:10.1093/infdis/jir734.

71. MacLean LM, Odiit M, Sternberg JM. Nitric oxide and cytokine synthesis in human African trypanosomiasis. J Infect Dis. 2001;184(8):1086-90.

72. Lejon V, Lardon J, Kenis G, Pinoges L, Legros D, Bisser S, et al. Interleukin (IL)-6, IL-8 and IL-10 in serum and CSF of Trypanosoma brucei gambiense sleeping sickness patients before and after treatment. Trans R Soc Trop Med Hyg. 2002;96(3):329-33.

73. Mabbott N, Coulson P, Smythies L, Wilson R, Sternberg J. African trypanosome infections in mice that lack the interferon-gamma receptor gene: nitric oxide-dependent and-independent suppression of T-cell proliferative responses and the development of anaemia. Immunology. 1998;94(4):476.

74. Olsson T, Bakhiet M, Edlund C, Höjeberg B, van der Meide PH, Kristensson K. Bidirectional activating signals between Trypanosoma brucei and CD8 + T cells: A trypanosome-released factor triggers interferon- $\gamma$ production that stimulates parasite growth. Eur J Immunol. 1991;21(10):2447-54.

75. Masocha W, Robertson B, Rottenberg ME, Mhlanga J, Sorokin L, Kristensson K. Cerebral vessel laminins and IFN- $\gamma$ define Trypanosoma brucei brucei penetration of the blood-brain barrier. J Clin Invest. 2004;114(5):689.

76. MacLean LM, Reiber H, Kennedy PG, Sternberg JM. Stage progression and neurological symptoms in Trypanosoma brucei rhodesiense sleeping sickness: role of the CNS inflammatory response. PLoS Negl Trop Dis. 2012;6(10):e1857. doi:10.1371/journal.pntd.0001857.

77. Pizarro TT, Cominelli F. Cloning IL-1 and the birth of a new era in cytokine biology. J Immunol. 2007;178(9):5411-2.

78. Schindler R, Mancilla J, Endres S, Ghorbani R, Clark SC, Dinarello CA. Correlations and interactions in the production of interleukin-6 (IL-6), IL-1, and tumor necrosis factor (TNF) in human blood mononuclear cells: IL-6 suppresses IL-1 and TNF. Blood. 1990;75(1):40-7.

79. Dinarello CA. Immunological and inflammatory functions of the interleukin-1 family. Annu Rev Immunol. 2009;27:519-50.

80. Ludigs K, Parfenov V, Du Pasquier RA, Guarda G. Type I IFN-mediated regulation of $\mathrm{IL}-1$ production in inflammatory disorders. Cell Mol Life Sci. 2012;69(20):3395-418.

81. Argaw AT, Zhang Y, Snyder BJ, Zhao ML, Kopp N, Lee SC, et al. IL-1 beta regulates blood-brain barrier permeability via reactivation of the hypoxia-angiogenesis program. J Immunol (Baltimore, Md: 1950). 2006;177(8):5574-84.

82. Quan N, He L, Lai W. Intraventricular infusion of antagonists of IL-1 and TNFa attenuates neurodegeneration induced by the infection of Trypanosoma brucei. J Neuroimmunol. 2003;138(1):92-8.

83. Quan N, Mhlanga JD, Whiteside MB, McCoy AN, Kristensson K, Herkenham M. Chronic overexpression of proinflammatory cytokines and histopathology in the brains of rats infected with Trypanosoma brucei. J Comp Neurol. 1999;414(1):114-30.

84. Wahl SM, McCartney-Francis N, Mergenhagen SE. Inflammatory and immunomodulatory roles of TGF- $\beta$. Immunol Today. 1989;10(8):258-61.
85. Espevik T, Figari IS, Shalaby MR, Lackides GA, Lewis GD, Shepard HM, et al. Inhibition of cytokine production by cyclosporin A and transforming growth factor $\beta$. J Exp Med. 1987;166(2):571-6.

86. Pedersen BK. The diseasome of physical inactivity —and the role of myokines in muscle_-fat cross talk. J Physiol. 2009;587(23):5559-68.

87. Kushima $Y$, Hatanaka H. Interleukin- 6 and leukemia inhibitory factor promote the survival of acetylcholinesterase-positive neurons in culture from embryonic rat spinal cord. Neurosci Lett. 1992;143(1):110-4.

88. Oka T, Oka K, Hosoi M, Hori T. Intracerebroventricular injection of interleukin-6 induces thermal hyperalgesia in rats. Brain Res. 1995;692(1):123-8.

89. Gadient RA, Otten UH. Interleukin-6 (IL-6) - a molecule with both beneficial and destructive potentials. Prog Neurobiol. 1997;52(5):379-90. doi:10.1016/S0301-0082(97)00021-X.

90. Brett FM, Mizisin AP, Powell HC, Campbell IL. Evolution of neuropathologic abnormalities associated with blood-brain barrier breakdown in transgenic mice expressing Interleukin-6 in astrocytes. J Neuropathol Exp Neurol. 1995;54(6):766-75

91. Nyawira-Maranga D, Kagira JM, Kinyanjui CK, Muturi Karanja S, Wangari Maina N, Ngotho M. IL-6 is upregulated in late-stage disease in monkeys experimentally infected with Trypanosoma brucei rhodesiense. Clin Dev Immunol. 2013;2013:320509. doi:10.1155/2013/320509.

92. MacLean LM, Odiit M, Sternberg JM. Intrathecal cytokine responses in Trypanosoma brucei rhodesiense sleeping sickness patients. Trans R Soc Trop Med Hyg. 2006;100(3):270-5.

93. Courtin D, Jamonneau V, Mathieu JF, Koffi M, Milet J, Yeminanga CS, et al. Comparison of cytokine plasma levels in human African trypanosomiasis. Trop Med Int Health. 2006;11(5):647-53.

94. Sabat R. IL-10 family of cytokines. Cytokine Growth Factor Rev. 2010;21(5):315-24.

95. Rousset F, Garcia E, Defrance T, Peronne C, Vezzio N, Hsu D-H, et al. Interleukin 10 is a potent growth and differentiation factor for activated human B lymphocytes. Proc Natl Acad Sci USA. 1992;89(5):1890-3.

96. Hunt NH, Grau GE. Cytokines: accelerators and brakes in the pathogenesis of cerebral malaria. Trends Immunol. 2003;24(9):491-9.

97. Sarciron M, Gherardi A. Cytokines involved in Toxoplasmic encephalitis. Scand J Immunol. 2000;52(6):534-43.

98. Atkinson AJ, Colburn WA, DeGruttola VG, DeMets DL, Downing GJ, Hoth $D F$, et al. Biomarkers and surrogate endpoints: preferred definitions and conceptual framework. Clin Pharmacol Ther. 2001;69(3):89-95.

99. Kennedy PG. Difficulties in diagnostic staging of human African trypanosomiasis. J Neuroparasitol. 2011;2:1-3.

100. Tiberti N, Matovu E, Hainard A, Enyaru JC, Lejon V, Robin X, et al. New biomarkers for stage determination in Trypanosoma brucei rhodesiense sleeping sickness patients. Clin Transl Med. 2013;2(1):1. doi:10.1186/2001-1326-2-1.

101. Lejon V, Roger I, Ngoyi DM, Menten J, Robays J, N'Siesi FX, et al. Novel markers for treatment outcome in late-stage Trypanosoma brucei gambiense trypanosomiasis. Clin Infect Dis. 2008;47(1):15-22.

102. Kato CD, Alibu VP, Nanteza A, Mugasa CM, Matovu E. Interleukin (IL)-6 and IL-10 are up regulated in late stage Trypanosoma brucei rhodesiense sleeping sickness. PLoS Negl Trop Dis. 2015;9(6):e0003835. doi:10.1371/ journal.pntd.0003835.

103. Courtioux B, Pervieux L, Vatunga G, Marin B, Josenando T, JauberteauMarchan MO, et al. Increased CXCL-13 levels in human African trypanosomiasis meningo-encephalitis. Trop Med Int Health. 2009;14(5):529-34.

104. Hainard A, Tiberti N, Robin X, Ngoyi DM, Matovu E, Enyaru JC, et al. Matrix metalloproteinase- 9 and intercellular adhesion molecule 1 are powerful staging markers for human African trypanosomiasis. Trop Med Int Health. 2011;16(1):119-26.

105. Tiberti N, Hainard A, Lejon V, Courtioux B, Matovu E, Enyaru JC, et al. Cerebrospinal fluid neopterin as marker of the meningo-encephalitic stage of Trypanosoma brucei gambiense sleeping sickness. PLoS One. 2012;7(7):e40909.

106. Tiberti N, Hainard A, Lejon V, Robin X, Ngoyi DM, Turck N, et al. Discovery and verification of osteopontin and $\beta$-2-microglobulin as promising markers for staging human African trypanosomiasis. Mol Cell Proteomics. 2010;9(12):2783-95.

107. Sternberg JM, Mitchell JA. Plasma neuronal specific enolase: a potential stage diagnostic marker in human African trypanosomiasis. Trans R Soc Trop Med Hyg. 2014;108(7):449-52. doi:10.1093/trstmh/tru065. 
108. da Costa AG, Antonelli LR, Costa PA, Pimentel JP, Garcia NP, Tarrago AM, et al. The robust and modulated biomarker network elicited by the Plasmodium vivax infection is mainly mediated by the IL-6/IL-10 axis and is associated with the parasite load. J Immunol Res. 2014;2014:318250. doi:10.1155/2014/318250.
109. Lucas R, Magez S, Songa B, Darji A, Hamers R, De Baetselier P. A role for TNF during African trypanosomiasis: involvement in parasite control immunosuppression and pathology. Res Immunol. 1993;144(5):370-6.
Submit your next manuscript to BioMed Central and we will help you at every step:

- We accept pre-submission inquiries

- Our selector tool helps you to find the most relevant journal

- We provide round the clock customer support

- Convenient online submission

- Thorough peer review

- Inclusion in PubMed and all major indexing services

- Maximum visibility for your research

Submit your manuscript at www.biomedcentral.com/submit
(OioMed Central 